\title{
Identification of disease-associated pathways in pancreatic cancer by integrating genome-wide association study and gene expression data
}

\author{
JIN LONG, ZHE LIU, XINGDA WU, YUANHONG XU and CHUNLIN GE
}

Department of General Surgery, The First Hospital of China Medical University, Shenyang, Liaoning 110001, P.R. China

Received April 1, 2015; Accepted May 17, 2016

DOI: $10.3892 / \mathrm{ol} .2016 .4637$

\begin{abstract}
In order to additionally understand the pathogenesis of pancreatic cancer (PC), the present study conducted pathway analysis based on genome-wide association study (GWAS) and gene expression data to predict genes that are associated with PC. GWAS data (accession no., pha002874.1) were downloaded from National Center for Biotechnology Information (NCBI) database of Genotypes and Phenotypes, which included data concerning 1,896 patients with PC and 1,939 control individuals. Gene expression data [accession no., GSE23952; human pancreatic carcinoma Panc-1 transforming growth factor- $\beta$ (TGF- $\beta$ ) treatment assay] were downloaded from NCBI Gene Expression Omnibus. Gene set enrichment analysis was used to identify significant pathways in the GWAS or gene expression profiles. Meta-analysis was performed based on pathway analysis of the two data sources. In total, 58 and 280 pathways were identified to be significant in the GWAS and gene expression data, respectively, with 7 pathways significant in both the data profiles. Hsa 04350 TGF- $\beta$ signaling pathway had the smallest meta P-value. Other significant pathways in the two data sources were negative regulation of DNA-dependent transcription, the nucleolus, negative regulation of RNA metabolic process, the cellular defense response, exocytosis and galactosyltransferase activity. By constructing the gene-pathway network, 5 pathways were closely associated, apart from exocytosis and galactosyltransferase activity pathways. Among the 7 pathways, 11 key genes ( $2.9 \%$ out of a total of 380 genes) from the GWAS data and 43 genes (10.5\% out of a total of 409 genes) from the gene expression data were differentially expressed. Only Abelson murine leukemia viral oncogene homolog 1 from the nucleolus pathway was significantly expressed in by both data sources. Overall, the results of the present analysis provide possible factors for the occurrence of PC, and the identification of the
\end{abstract}

Correspondence to: Mr. Jin Long, Department of General Surgery, The First Hospital of China Medical University, 155 Nanjingbei Street, Shenyang, Liaoning 110001, P.R. China

E-mail: jinlongdjdds@163.com

Key words: pancreatic cancer, GWAS, gene expression profile, meta-analysis, TGF- $\beta$ signaling pathway, $A B L 1$ pathways and genes associated with $\mathrm{PC}$ provides valuable data for investigating the pathogenesis of $\mathrm{PC}$ in future studies.

\section{Introduction}

Pancreatic cancer (PC; OMIM, 260350) is a highly lethal disease; it is one of the deadliest cancers worldwide, with a mortality rate of $99 \%$ and 5-year relative survival rate of $<5 \%$ (1), and almost all patients with PC develop metastasis. The etiology of $\mathrm{PC}$ remains elusive; smoking is the best known risk factor (2).

Advances in molecular biology have greatly improved understanding the pathogenesis of PC. The development of $\mathrm{PC}$ requires the transformation of normal pancreatic cells to precursor pancreatic intraepithelial neoplasia, which is associated with gene mutations, continuous alterations in nuclei, loss of polarity and alterations to the architecture of cells (3). In addition, chromosome abnormalities are involved in the pathophysiology and development of PC, which usually presents as a loss or gain of alleles in various chromosomes (4). It has been reported that the development and progression of PC is caused by activation of oncogenes and inactivation of tumor suppressor genes, as well as deregulation of numerous signaling pathways, including epidermal growth factor receptor, protein kinase B and nuclear factor kappa B pathways (5). In addition, Hedgehog signaling pathway, an essential pathway during embryonic pancreatic development, is involved in several types of cancer and may be an important mediator in human PC (6). Previous studies indicate that $\mathrm{PC}$ has a complex genomic landscape with frequent copy number alterations and point mutations (7). It has been demonstrated that common mutated genes in PC include Kirsten rat sarcoma viral oncogene homolog (K-ras; 74-100\%), p16INK4a ( $\leq 98 \%)$, p53 (43-76\%), deleted in pancreatic cancer, locus $4(\sim 50 \%)$, human epidermal growth factor (HER)-2/neu ( 65\%) and Fragile Histidine Triad ( 70\%) (8-12); $K$-ras and HER-2/neu are proto-oncogenes, while all the other genes are tumor suppressor genes (7). Through comprehensive genetic analysis of 24 samples of PC, Jones et al (13) demonstrated that PC contained an average of 63 genetic alterations, the majority of which were point mutations, and these alterations defined a core set of 12 cellular signaling pathways, which were genetically altered in $67-100 \%$ of PC tumors. Additionally, Biankin et al (14) defined 16 significant mutated genes, reaffirmed known mutations [K-RAS, tumor 
protein p53, cyclin-dependent kinase inhibitor 2A, SMAD4, myeloid/lymphoid or mixed-lineage leukemia 3, transforming growth factor, beta receptor II, AT-rich interaction domain (ARID) 1A and splicing factor $3 \mathrm{~b}$ subunit 1] and uncovered novel mutated genes, including genes involved in chromatin modification (enhancer of polycomb homolog 1 and ARID2), DNA damage repair (ATM serine/threonine kinase) and other mechanisms in axon guidance (zinc finger imprinted 2, mitogen-activated protein kinase kinase 4 , sodium leak channel, non-selective, solute carrier family 16 member 4 and MAGEA6). In a humanized genetically modified mouse model of pancreatic ductal adenocarcinoma, which accounts for $>90 \%$ of PC, Rosenfeldt et al (15) revealed that loss of autophagy did not block tumor progression, but actually accelerated tumor onset.

Genome-wide association study (GWAS) aims to detect variants at genomic loci associated with complex traits in a population and, in particular, detect associations between common single-nucleotide polymorphisms (SNPs) and common diseases (16). Gene expression is another source of gene data for investigating complex genetic disease, which describes the type and abundance of gene expression in specific cells or tissues under certain conditions (17). Gene Expression Omnibus Series (GSE) dataset GSE 23952 [human pancreatic carcinoma Panc- 1 transforming growth factor- $\beta$ (TGF- $\beta$ ) treatment assay] was used by the present study, which has also been used by previous studies. Kato et al (18) analyzed two datasets (GSE 17708 and 23952) to identify genes encoding secreted proteins on GenePattern. Xu and Liu (19) used several datasets to study the aberrant expression of cytoplasmic polyadenylation element binding protein 4 . Additionally, Gröger (20) developed a comprehensive meta-analysis combining 24 epithelial mesenchymal transition (EMT) datasets, including GSE 23952, to investigate the effectors of EMT. However, none of these studies focussed on pathway analysis in $\mathrm{PC}$.

The present study combined GWAS and gene expression data to identify important pathways for the pathogenesis of PC. Gene set enrichment analysis (GSEA) was used to identify over-represented pathways in GWAS or gene expression profiles. Meta-analysis was performed to select significant pathways in GWAS and gene expression data.

\section{Materials and methods}

GWAS and gene expression profile. GWAS data (accession no., pha002874.1) were downloaded from National Center for Biotechnology Information (NCBI) database of Genotypes and Phenotypes (www.ncbi.nlm.nih. gov/projects/SNP/gViewer/gView.cgi? aid=2874). The data was obtained by genotyping with the Illumina Hap 500 Infinium genotyping assay (Illumina, Inc., San Diego, CA, USA) on 1,896 PC patients and 1,939 control individuals drawn from 12 prospective cohorts plus one hospital-based case-control study (21). A total of 522,293 SNPs were used in this analysis.

For gene expression analysis, the present study utilized the microarray data set submitted by Maupin et al (22). The data were downloaded from NCBI Gene Expression Omnibus (www.ncbi.nlm.nih.gov/sites/GDSbrowser?acc=GDS4106; accession no., GSE23952; human pancreatic carcinoma Panc-1
TGF- $\beta$ treatment assay). In the study by Maupin et al, the human pancreatic adenocarcinoma Panc-1 cell line was treated with TGF- $\beta$ to induce EMT, and the study was repeated three times. Samples were assayed using Affymetrix Human Genome U133 Plus 2.0 Array (Affymetrix, Santa Clara, CA, USA), and probes with the largest differential expression value were selected. A total of 54,623 probe-sets were obtained following normalization. Differentially expressed genes (DEGs) derived from probes were used for further analysis.

GWAS data analysis: Mapping SNPs to genes. SNPs in the GWAS data were mapped to corresponding genes. The SNPs were annotated based on hg19 (hgdownload.cse.ucsc.edu/goldenPath/hg19/bigZips/). Genes were identified according to their sitting priority [exon region $>$ intron region $>5$ ' untranslated region (UTR) > 3' UTR]. If there were no genes identified in any genetic locus, genes closest to one side of the SNP were included. If more than one SNP was mapped to a gene, genes with the smallest P-value were selected in from the GWAS data.

GSEA pathway analysis. Pathway analysis was performed using GSEA on GWAS and gene expression profile data. GSEA statistically tests whether members of a predefined gene set are randomly distributed throughout a ranked list of genes or whether the members of the gene set cluster toward the top of the list provided by the Broad Institute (www.broadinstitute. org/gsea/index.jsp) (23-25). Pathway analysis of gene sets was performed through Gene Ontology (GO) pathways (c5.all. v4.0.symbols.gmt) from the Molecular Signatures Database (www.broadinstitute.org/msigdb) (23) and Kyoto Encyclopedia of Genes and Genomes (KEGG) pathways (www.genome. $\mathrm{jp} / \mathrm{kegg} /)(26)$.

Meta-analysis. Pathways with a significant difference in GWAS and gene expression data were selected to perform meta-analysis. Meta P-values were obtained by Fisher's combined probability test (27). The combined P-value was calculated by adding $-2 \ln$ (P-value) of the two tests for a pathway. Subsequently, a $\chi^{2}$-test distribution was performed, which was used to determine the meta $\mathrm{P}$-value (28). $\mathrm{P}<0.05$ was considered to indicate a statistically significant difference. All statistical tests were performed using Perl language version 5.24.0 (www.perl.org/).

\section{Results}

Meta-analysis of over-represented pathways in $P C$. The 522,293 SNPs loci were contained in GWAS data and were mapped to 18,910 genes. In total, 58 over-represented pathways were selected by GSEA pathway analysis. For gene expression data, 54,623 probes were obtained and the probes were mapped to 31,620 genes. Subsequently, 230 pathways were identified by GSEA $(\mathrm{P}<0.05)$.

A meta-analysis of over-represented pathways was performed to identify statistically significant pathways in the combined GWAS and gene expression PC data. A total of 7 over-represented pathways from the GWAS and gene expression data were identified (Fig. 1; Table I), of which 6 pathways were GO signal pathways, and 1 pathway (KEGG ID, hsa 04350) 
was from the KEGG database (Fig. 2). Additionally, a TGF- $\beta$ signaling pathway (hsa 04350) had the smallest meta P-value. In this signaling pathway, transcription factor Dp-1 (TFDP1), activin A receptor type (ACVR) $2 \mathrm{~A}$ and v-myc avian myelocytomatosis viral oncogene homolog $(M Y C)$ were differentially expressed in GWAS data, while noggin $(N O G)$, inhibitor of DNA binding 1, HLH protein (IDI), left-right determination factor 1 (LEFTY1) and ACVR1 were differentially expressed in gene expression data. The other significant pathways in the two data sources were as follows: Negative regulation of DNA-dependent transcription (GO: 0045892); the nucleolus (GO: 0005730); negative regulation of RNA metabolic process (GO: 0051253); the cellular defense response (GO: 0006968); exocytosis (GO: 0006887); and galactosyltransferase activity (GO: 0008378).

According to the constructed gene-pathway network, exocytosis (GO: 0006887) and galactosyltransferase activity (GO: 0008378) had no connection with the other pathways, while the other 5 pathways were closely associated with each other (Fig. 1). As shown in the network, the TGF- $\beta$ signaling pathway (hsa 04350) and nucleolus pathway (GO: 0005730) were closely connected, with numerous genes overlapping each other, as well as negative regulation of RNA metabolic process pathway (GO: 0051253) and negative regulation of DNA-dependent transcription pathway (GO: 0045892). Additionally, 4 pathways, including negative regulation of RNA metabolic process (GO: 0051253), negative regulation of DNA-dependent transcription (GO: 0045892), the nucleolus (GO: 0005730) and TGF- $\beta$ signaling pathway (hsa 04350), were connected via recombination signal binding protein for immunoglobulin kappa $\mathrm{J}$ region (RBPJ) and MDM2 proto-oncogene (MDM2), while cellular defense response (GO: 0006968), negative regulation of RNA metabolic process (GO: 0051253) and negative regulation of DNA-dependent transcription (GO: 0045892) were associated via SMAD2, SMAD3, SMAD4, bone morphogenetic protein $(B M P) 2$ and follistatin.

Identification of key genes for PC pathways. To identify specific genes within the 7 pathways identified by the present study, individual genes with $\mathrm{P}<0.05$ were selected from GWAS and gene expression data. Among the 7 pathways, 11 key genes (2.9\% out of a total of 380 genes) from GWAS data were differentially expressed (Table II), including neuroligin 1 , regulating synaptic membrane exocytosis 1 , protein phosphatase 2 regulatory subunit $2 C, B M P 6$, zinc finger protein 238 , Kruppel-like factor 4, MYB Binding Protein (P160) 1a, ABL proto-oncogene 1 , non-receptor tyrosine kinase $(A B L 1)$, ribosomal protein L11, topoisomerase (DNA) I. For the gene expression data, 43 genes (10.5\% out of a total of 409 genes) were identified as being significantly expressed (Table III). Among these significant genes, only $A B L 1$ from the nucleolus pathway (GO: 0005730) was significantly expressed in both GWAS $\left[\mathrm{P}=0.002085 ; \mathrm{P}\right.$-value $<\min \left(0.5^{*} \mathrm{~N}\right)$, where $\mathrm{N}=$ gene number] and gene expression data [value $=1.7271$; value $<\max$ $\left.\left(0.5^{*} \mathrm{~N}\right)\right]$

\section{Discussion}

According to meta-analysis based on GSEA pathway analysis, 7 pathways were identified by the present study to
Table I. A total of 7 pathways were determined by meta-analysis using combined genome-wide association study and gene expression data.

\begin{tabular}{llc}
\hline Pathway & \multicolumn{1}{c}{ Term } & Meta P-value \\
\hline Hsa 04350 & TGF- $\beta$ signaling pathway & 0.00037 \\
GO: 0005730 & Nucleolus & 0.00045 \\
GO: 0006968 & Cellular defense response & 0.00145 \\
GO: 0008378 & Galactosyltransferase activity & 0.00182 \\
GO: 0006887 & Exocytosis & 0.00219 \\
GO: 0051253 & Negative regulation of RNA & 0.00331 \\
& metabolic process & \\
GO: 0045892 & Negative regulation of DNA- & \\
& dependent transcription & 0.00333 \\
\hline
\end{tabular}

TGF- $\beta$, transforming growth factor- $\beta$.

Table II. DEGs from genome-wide association study data of each pathway identified by meta-analysis.

\begin{tabular}{llc}
\hline Pathway & \multicolumn{1}{c}{ DEGs } & P-value \\
\hline GO: 0008378 & - & 0.005 \\
GO: 0051253 & ZNF238, KLF4 & 0.031 \\
Hsa 04350 & $P P P 2 R 2 C, B M P 6, T F D P 1, A C V R 2 A$, & 0.033 \\
& $P P P 2 R 2 A, B M P 7, M Y C$ & \\
GO: 0045892 & ZNF238, KLF4 & 0.034 \\
GO: 0006968 & - & 0.037 \\
GO: 0006887 & NLGN1, RIMS1 & 0.039 \\
GO: 0005730 & $M Y B B P 1 A, A B L 1, R P L 11, T O P 1$ & 0.040 \\
\hline
\end{tabular}

DEGs, differentially expressed genes; -, no genes differentially expressed in the pathway.

be significant in GWAS and gene expression profiles. These pathways were associated with the TGF- $\beta$ signaling pathway (hsa 04350), negative regulation of DNA-dependent transcription (GO: 0045892), the nucleolus (GO: 0005730), negative regulation of RNA metabolic process (GO: 0051253), the cellular defense response (GO: 0006968), exocytosis (GO: 0006887) and galactosyltransferase activity (GO: 0008378). The TGF- $\beta$ signaling pathway had the smallest meta P-value. By constructing the gene-pathway network, 5 pathways were identified as closely connected, apart from exocytosis and galactosyltransferase activity pathways. Among the 7 pathways, 11 key genes (2.9\% out of a total of 380 genes) from GWAS data and 43 genes ( $10.5 \%$ out of a total of 409 genes) from gene expression data were differentially expressed. Only $A B L 1$ from the nucleolus pathway was significantly expressed in the both data sources.

In total, 3 genes (TFDP1, ACVR $2 A$ and $M Y C$ ) from GWAS data and 4 genes (NOG, IDI, LEFTY1 and ACVRI) from gene expression profile were differentially expressed in the TGF- $\beta$ signaling pathway. TGF- $\beta$ family members include TGF-betas, activins and BMPs, which are 


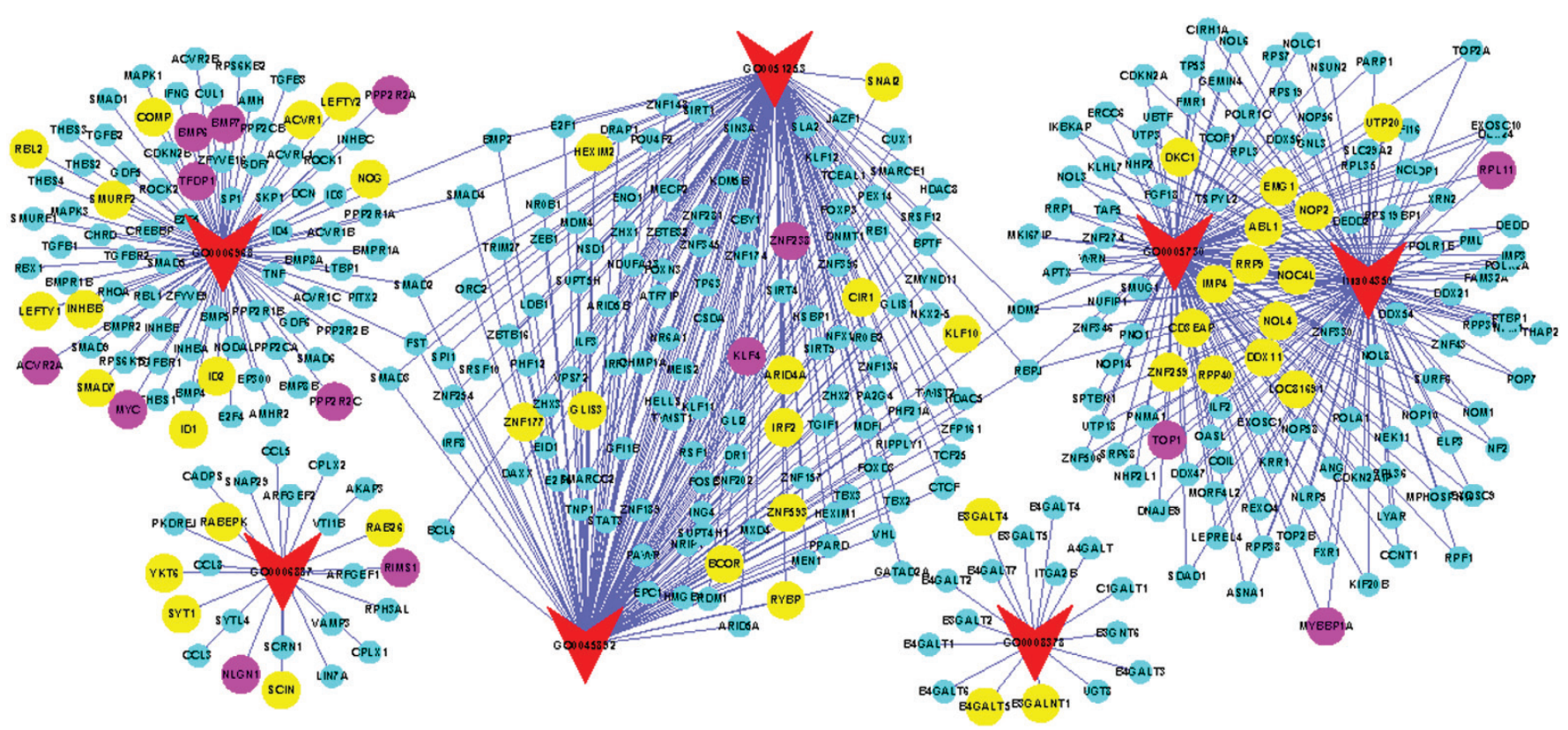

Figure 1. Gene-pathway network. Red arrows indicate signaling pathways; purple circles indicate genes differentially expressed in GWAS data; yellow circles indicate genes differentially expressed in gene expression profile; blue circles indicate normal genes in GWAS or gene expression data. GWAS, genome-wide association study.

structurally associated with secreted cytokines (29). In addition, the TGF- $\beta$ family regulates numerous cellular processes, including cell proliferation, recognition, differentiation and apoptosis (30). MYC, an over-expressed proto-oncogene in the TGF- $\beta$ signaling pathway, encodes a DNA-binding factor that activates or represses transcription (31). Via this mechanism, MYC regulates the expression of numerous target genes, which control key cellular functions, such as cell growth and cell cycle progression (32). Therefore, deregulated $M Y C$ expression, resulting from various types of genetic alterations, results in constitutive $M Y C$ activity in a variety of cancers and promotes oncogenesis (33). If PC cells are stably transfected with a dominant-negative mutant of $M Y C$ (c-Myc), their proliferation is markedly inhibited (34). Grippo et al (35) studied myc-associated acinar-to-ductal metaplasia in Ela-c-myc transgenic rats, and demonstrated that c-myc was associated with human pancreatic neoplasms, which was sufficient to induce acinar hyperplasia. Additionally, Köenig et al (36) revealed a novel mechanism regulating cell growth in PC: Serum promotes the occurrence of $\mathrm{PC}$ through the induction of proliferative NFAT/c-Myc axis by impaired c-Myc expression and reduces tumor growth upon nuclear factor of activated T-cells depletion in vitro and in vivo. TFDPl is the first member of the E2F transcription factor family that regulates the expression of various cellular promoters, particularly those involved in the cell cycle (37). Abba et al (38) identified that TFDP1 exhibits the highest frequency of amplification affecting primary breast cancer samples. In addition, meta-analysis reveals a strong association between a high expression of TFDP1 or $N O G$ and the decreased overall survival in patients with breast cancer (39). Furthermore, overexpression of TFDPI may contribute to the progression of certain hepatocellular carcinomas by promoting the growth of tumor cells (40). Additionally, IDI, a gene associated with cell growth, senescence, differentiation and angiogenesis, participates in numerous tumor processes $(41,42)$. Other genes, including $A C V R 2 A, L E F T Y 1$ and $A C V R 1$, are mostly associated with pituitary tumors (43), left-right axis malformations (44) and fibrodysplasia ossificans progressive (45), respectively. These genes, including TFDP1, NOG, IDI ACVR2A, LEFTY1 and $A C V R 1$, were identified as being associated with $\mathrm{PC}$ in the present study, which is rarely reported in $\mathrm{PC}$ pathogenesis. Thus, further study is required verify these genes in a PC context.

According to the constructed gene-signal pathway network, 5 pathways were demonstrated to be closely connected in the present study, apart from exocytosis and galactosyltransferase activity pathway. RBPJ and $M D M 2$ were bridges that connected 4 of these pathways. Masui et al (46) demonstrated that pancreas specific transcription factor, 1a (PTFLA), a basic helix-loop-helix transcription factor required for pancreatic development, interacts with $R B P J$ within a stable trimeric DNA-binding complex, PTF1, during early PC development in mice. Introduction of a PTFLA mutant, which is unable to bind $R B P J$, truncated pancreatic development at an immature stage and acini or islets were not formed. MDM2 is an E3 ubiquitin ligase that targets the tumor suppressor p53 protein for proteasomal degradation. p53 induces cell cycle arrest or apoptosis in response to cellular stress (47). The MDM2 oncoprotein promotes cell survival and cell cycle progression by inhibiting the p53 tumor suppressor protein (48).

ABL1 (OMIM entry, *189980) was first identified as an oncogene from the $A B L$ family of nonreceptor tyrosine kinases, and it transduces diverse extracellular signals to protein networks that control proliferation, survival, migration and invasion of cells (49). ABL1 encodes a cytoplasmic and nuclear protein tyrosine kinase, which is involved in cell differentiation, division and adhesion, and the stress response (50). 
Table III. DEGs from gene expression profiles of each pathway identified by meta-analysis.

\begin{tabular}{lll}
\hline Pathway & \multicolumn{1}{c}{ DEGs } & P-value \\
\hline Hsa 04350 & NOG, ID1, LEFTY2, INHBB, LEFTY1, SMAD7, COMP, RBL2, ID2, SMURF2, ACVR1 \\
GO: 0005730 & CD3EAP, NOL4, IMP4, DDX11, ZNF259, EMG1, RRP9, ABL1, LOC81691, DKC1, RPP40, & 0.001 \\
& UTP20, NOC4L, NOP2 & 0.001 \\
GO: 0006968 & KLRC3, KLRC4,FOSL1, LGALS3BP, MICB \\
GO: 0006887 & RAB26, SYT1, RABEPK, YKT6, SCIN & 0.004 \\
GO: 0045892 & SNAI2, RYBP, GLIS3, IRF2, HEXIM2, KLF10, CIR1, BCOR, ZNF177, ZNF593, ARID4A \\
GO: 0051253 & SNAI2, RYBP, GLIS3, IRF2, HEXIM2, KLF10, CIR1, BCOR, ZNF177, ZNF593, ARID4A \\
GO: 0008378 & B3GALT4, B4GALT5, B3GALNT1 & 0.006 \\
\end{tabular}

DEGs, differentially expressed genes.

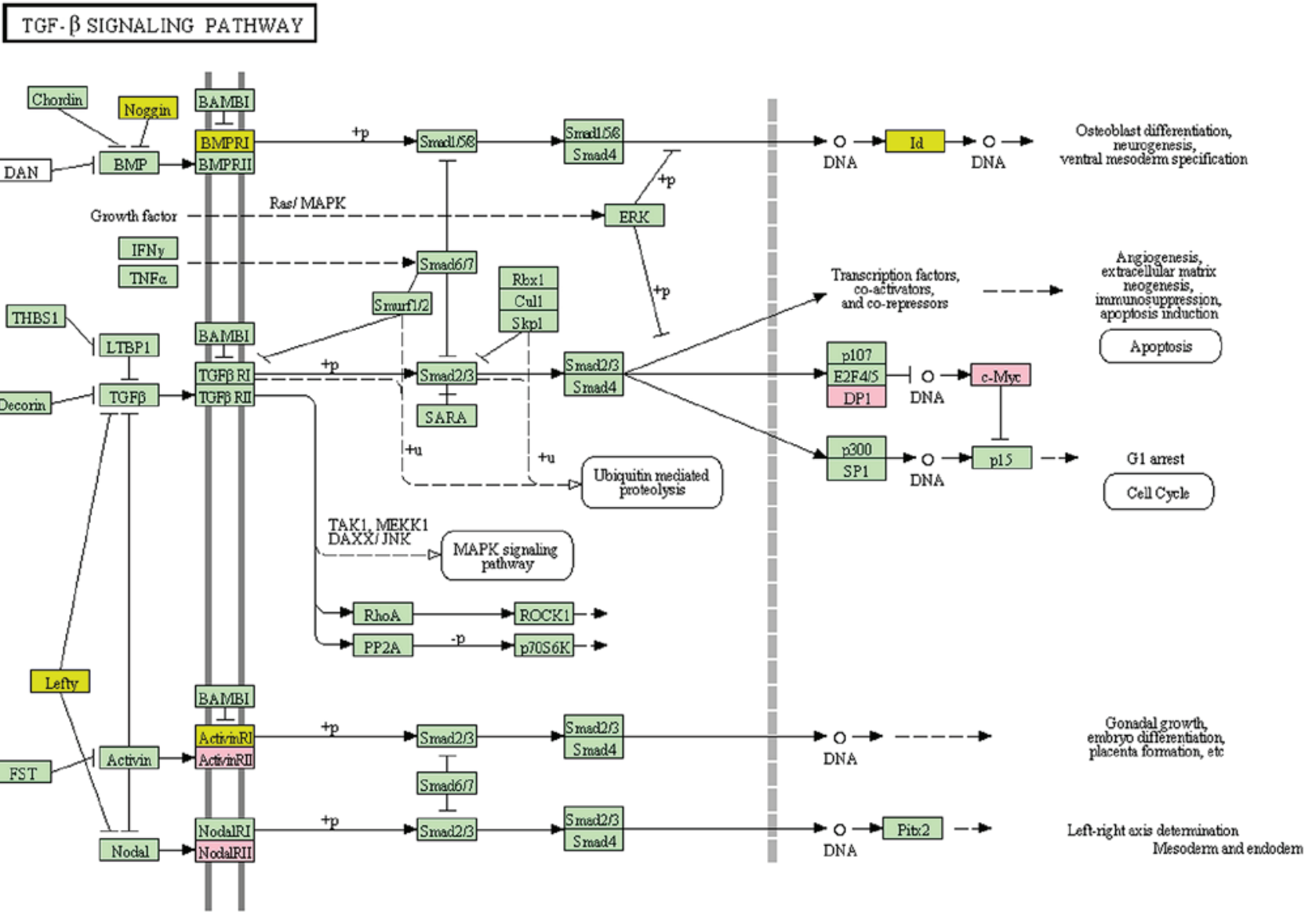

Figure 2. TGF- $\beta$ signaling pathway. Pink boxes indicate genes differentially expressed in genome-wide association study data; yellow boxes indicate genes differentially expressed in gene expression data; green boxes indicate normal genes in TGF- $\beta$ signaling pathways. TGF- $\beta$, transforming growth factor- $\beta$.

Alterations of $A B L 1$ by chromosomal rearrangement or viral transduction leads to malignant transformation (51). The overexpression of microRNA (miR)-203 leads to a poor survival of cancer, due to its oncogenic function; however, miR-203 exhibits tumor-suppressor qualities in PC by inhibiting the expression of $A B L 1$ and $B C R-A B L 1$, resulting in an inhibition of cell proliferation (52).

Overall, through the combined pathway analysis of GWAS and gene expression, 7 pathways were demonstrated to be significant by meta-analysis performed by the present study.
Among all the significantly expressed genes, only one gene, $A B L 1$, was differentially expressed in the both data sources. The present study identified $M Y C$ as the most probable gene associated with the TGF- $\beta$ signaling pathway in PC. In conclusion, the results of the present analysis provide possible factors for the occurrence of PC, and the identification of pathways and genes provides valuable data for investigating the pathogenesis of PC. However, bioinformatics analysis generally lacks experimental support, so additional study is required to verify the results of the present study. 


\section{Acknowledgements}

The authors wish to express their warm thanks to Fenghe Information Technology Co., Ltd (Shanghai, China), whose ideas and help provided valuable dimensions to the present study.

\section{References}

1. Thomas J, Kim M, Balakrishnan L, Nanjappa V, Raju R, Marimuthu A, Radhakrishnan A, Muthusamy B, Khan AA, Sakamuri S, et al: Pancreatic cancer database: An integrative resource for pancreatic cancer. Cancer Biol Ther 15: 963-967, 2014.

2. Lowenfels $A B$ and Maisonneuve P: Epidemiology and risk factors for pancreatic cancer. Best Pract Res Clin Gastroenterol 20: 197-209, 2006.

3. Hruban RH, Adsay NV, Albores-Saavedra J, Compton C, Garrett ES, Goodman SN, Kern SE, Klimstra DS, Klöppel G, Longnecker DS, et al: Pancreatic intraepithelial neoplasia: A new nomenclature and classification system for pancreatic duct lesions. Am J Surg Pathol 25: 579-586, 2001.

4. Griffin CA, Hruban RH, Long PP, Morsberger LA, Douna-Issa F and Yeo CJ: Chromosome abnormalities in pancreatic adenocarcinoma. Genes Chromosomes Cancer 9: 93-100, 1994.

5. Sarkar FH, Banerjee S and Li Y: Pancreatic cancer: Pathogenesis, prevention and treatment. Toxicol Appl Pharmacol 224: 326-336, 2007.

6. Thayer SP, di Magliano MP, Heiser PW, Nielsen CM, Roberts DJ, Lauwers GY, Qi YP, Gysin S, Fernández-del Castillo C, Yajnik V, et al: Hedgehog is an early and late mediator of pancreatic cancer tumorigenesis. Nature 425: 851-856, 2003

7. Strimpakos A, Saif MW and Syrigos KN: Pancreatic cancer: From molecular pathogenesis to targeted therapy. Cancer Metastasis Rev 27: 495-522, 2008

8. Hingorani SR, Petricoin EF, Maitra A, Rajapakse V, King C, Jacobetz MA, Ross S, Conrads TP, Veenstra TD, Hitt BA, et al: Preinvasive and invasive ductal pancreatic cancer and its early detection in the mouse. Cancer Cell 4: 437-450, 2003.

9. Goldstein AM, Fraser MC, Struewing JP, Hussussian CJ, Ranade K, Zametkin DP, Fontaine LS, Organic SM, Dracopoli NC, Clark WH Jr, et al: Increased risk of pancreatic cancer in melanoma-prone kindreds with p16INK4 mutations. N Engl J Med 333: 970-975, 1995.

10. Pellegata N, Sessa F, Renault B, Bonato M, Leone BE, Solcia E and Ranzani GN: K-ras and p53 gene mutations in pancreatic cancer: Ductal and nonductal tumors progress through different genetic lesions. Cancer Res 54: 1556-1560, 1994.

11. Grau AM, Zhang L, Wang W, Ruan S, Evans DB, Abbruzzese JL, Zhang W and Chiao PJ: Induction of p21waf1 expression and growth inhibition by transforming growth factor beta involve the tumor suppressor gene DPC4 in human pancreatic adenocarcinoma cells. Cancer Res 57: 3929-3934, 1997.

12. Sorio C, Baron A, Orlandini S, Zamboni G, Pederzoli P, Huebner K and Scarpa A: The FHIT gene is expressed in pancreatic ductular cells and is altered in pancreatic cancers. Cancer Res 59: 1308-1314, 1999.

13. Jones S, Zhang X, Parsons DW, Lin JC, Leary RJ, Angenendt P, Mankoo P, Carter H, Kamiyama H, Jimeno A, et al: Core signaling pathways in human pancreatic cancers revealed by global genomic analyses. Science 321: 1801-1806, 2008.

14. Biankin AV, Waddell N, Kassahn KS, Gingras MC, Muthuswamy LB, Johns AL, Miller DK, Wilson PJ, Patch AM, $\mathrm{Wu} \mathrm{J}$, et al: Pancreatic cancer genomes reveal aberrations in axon guidance pathway genes. Nature 491: 399-405, 2012.

15. Rosenfeldt MT, O'Prey J, Morton JP, Nixon C, MacKay G, Mrowinska A, Au A, Rai TS, Zheng L, Ridgway R, et al: p53 status determines the role of autophagy in pancreatic tumour development. Nature 504: 296-300, 2013.

16. Cantor RM, Lange K and Sinsheimer JS: Prioritizing GWAS results: A review of statistical methods and recommendations for their application. Am J Hum Genet 86: 6-22, 2010.

17. Croteau-Chonka DC, Qiu W, Carey VJ, Weiss ST and Raby BA: Gene set enrichment analyses of gene expression associations with asthma control hint at candidate drug pathways. Am J Respir Crit Care Med 189: A5274, 2014.
18. Kato S, Hayakawa Y, Sakurai H, Saiki I and Yokoyama S: Mesenchymal-transitioned cancer cells instigate the invasion of epithelial cancer cells through secretion of WNT3 and WNT5B. Cancer Sci 105: 281-289, 2014.

19. Xu H and Liu B: CPEB4 is a candidate biomarker for defining metastatic cancers and directing personalized therapies. Med Hypotheses 81: 875-877, 2013.

20. Gröger C: The role of Neuropilin 2 in hepatocellular carcinoma: From meta-analysis to target characterization. Journal, 2013.

21. Amundadottir L, Kraft P, Stolzenberg-Solomon RZ, Fuchs CS, Petersen GM, Arslan AA, Bueno-de-Mesquita HB, Gross M, Helzlsouer K, Jacobs EJ, et al: Genome-wide association study identifies variants in the ABO locus associated with susceptibility to pancreatic cancer. Nat Genet 41: 986-990, 2009.

22. Maupin KA, Sinha A, Eugster E, Miller J, Ross J, Paulino V, Keshamouni VG, Tran N, Berens M, Webb C and Haab BB: Glycogene expression alterations associated with pancreatic cancer epithelial-mesenchymal transition in complementary model systems. PloS One 5: e13002, 2010.

23. Subramanian A, Tamayo P, Mootha VK, Mukherjee S, Ebert BL, Gillette MA, Paulovich A, Pomeroy SL, Golub TR, Lander ES and Mesirov JP: Gene set enrichment analysis: A knowledge-based approach for interpreting genome-wide expression profiles. Proc Natl Acad Sci USA 102: 15545-15550, 2005.

24. Joslyn G, Ravindranathan A, Brush G, Schuckit M and White RL: Human variation in alcohol response is influenced by variation in neuronal signaling genes. Alcohol Clin Exp Res 34: 800-812, 2010.

25. Mootha VK, Lindgren CM, Eriksson KF, Subramanian A Sihag S, Lehar J, Puigserver P, Carlsson E, Ridderstråle M, Laurila E, et al: PGC-1alpha-responsive genes involved in oxidative phosphorylation are coordinately downregulated in human diabetes. Nat Genet 34: 267-273, 2003.

26. Kanehisa M, Goto S, Sato Y, Furumichi M and Tanabe M: KEGG for integration and interpretation of large-scale molecular data sets. Nucleic Acids Res 40 (Database Issue): D109-D114, 2012.

27. Rice WR: A consensus combined P-value test and the family-wide significance of component tests. Biometrics 303-308, 1990.

28. Edwards YJ, Beecham GW, Scott WK, Khuri S, Bademci G, Tekin D, Martin ER, Jiang Z, Mash DC, ffrench-Mullen J, et al: Identifying consensus disease pathways in Parkinson's disease using an integrative systems biology approach. PLoS One 6: e16917, 2011.

29. Unsicker K, Spittau B and Krieglstein K: The multiple facets of the TGF- $\beta$ family cytokine growth/differentiation factor-15/macrophage inhibitory cytokine-1. Cytokine Growth Factor Rev 24: 373-384, 2013.

30. Shi Y and Massagué J: Mechanisms of TGF-beta signaling from cell membrane to the nucleus. Cell 113: 685-700, 2003.

31. Murre C, McCaw PS and Baltimore D: A new DNA binding and dimerization motif in immunoglobulin enhancer binding, daughterless, MyoD, and myc proteins. Cell 56: 777-783, 1989.

32. Dang CV: MYC on the path to cancer. Cell 149: 22-35, 2012.

33. Dominguez-Sola D, Ying CY, Grandori C, Ruggiero L, Chen B, Li M, Galloway DA, Gu W, Gautier J and Dalla-Favera R: Non-transcriptional control of DNA replication by c-Myc. Nature 448: 445-451, 2007.

34. Asano T, Yao Y, Zhu J, Li D, Abbruzzese JL and Reddy SA: The PI 3-kinase/Akt signaling pathway is activated due to aberrant Pten expression and targets transcription factors NF-kappaB and c-Myc in pancreatic cancer cells. Oncogene 23: 8571-8580, 2004.

35. Grippo PJ and Sandgren EP: Acinar-to-ductal metaplasia accompanies c-myc-induced exocrine pancreatic cancer progression in transgenic rodents. Int J Cancer 131: 1243-1248, 2012.

36. Köenig A, Linhart T, Schlengemann K, Reutlinger K, Wegele J, Adler G, Singh G, Hofmann L, Kunsch S, Büch T, et al: NFAT-induced histone acetylation relay switch promotes c-Myc-dependent growth in pancreatic cancer cells. Gastroenterology 138: 1189-1199.e1-e2, 2010.

37. Calzone L, Gelay A, Zinovyev A, Radvanyi F and Barillot E: A comprehensive modular map of molecular interactions in RB/E2F pathway. Mol Syst Biol 4: 173, 2008.

38. Abba MC, Fabris VT, Hu Y, Kittrell FS, Cai WW, Donehower LA, Sahin A, Medina D and Aldaz CM: Identification of novel amplification gene targets in mouse and human breast cancer at a syntenic cluster mapping to mouse ch8A1 and human ch13q34. Cancer Res 67: 4104-4112, 2007.

39. Tarragona M, Pavlovic M, Arnal-Estapé A, Urosevic J, Morales M, Guiu M, Planet E, González-Suárez E and Gomis RR: Identification of NOG as a specific breast cancer bone metastasis-supporting gene. J Biol Chem 287: 21346-21355, 2012. 
40. Yasui K, Okamoto H, Arii S and Inazawa J: Association of over-expressed TFDP1 with progression of hepatocellular carcinomas. J Hum Genet 48: 609-613, 2003.

41. Kebebew E, Peng M, Treseler PA, Clark OH, Duh QY, Ginzinger D and Miner R: Id1 gene expression is up-regulated in hyperplastic and neoplastic thyroid tissue and regulates growth and differentiation in thyroid cancer cells. J Clin Endocrinol Metab 89: 6105-6111, 2004.

42. Gumireddy K, Li A, Gimotty PA, Klein-Szanto AJ, Showe LC, Katsaros D, Coukos G, Zhang L and Huang Q: KLF17 is a negative regulator of epithelial-mesenchymal transition and metastasis in breast cancer. Nat Cell Biol 11: 1297-1304, 2009.

43. D'Abronzo FH, Swearingen B, Klibanski A and Alexander JM: Mutational Analysis of activin/transforming growth factor-beta type I and type II receptor kinases in human pituitary tumors. J Clin Endocrinol Metab 84: 1716-1721, 1999.

44. Casey B and Hackett BP: Left-right axis malformations in man and mouse. Curr Opin Genet Dev 10: 257-261, 2000.

45. Shore EM, Xu M, Feldman GJ, Fenstermacher DA, Cho TJ, Choi IH, Connor JM, Delai P, Glaser DL, LeMerrer M, et al: A recurrent mutation in the BMP type I receptor ACVR1 causes inherited and sporadic fibrodysplasia ossificans progressiva. Nat Genet 38: 525-527, 2006.
46. Masui T, Long Q, Beres TM, Magnuson MA and MacDonald RJ: Early pancreatic development requires the vertebrate Suppressor of Hairless (RBPJ) in the PTF1 bHLH complex. Genes Dev 21: 2629-2643, 2007.

47. Sasaki M, Kawahara K, Nishio M, Mimori K, Kogo R, Hamada K, Itoh B, Wang J, Komatsu Y, Yang YR, et al: Regulation of the MDM2-P53 pathway and tumor growth by PICT1 via nucleolar RPL11. Nat Med 17: 944-951, 2011.

48. Mayo LD and Donner DB: A phosphatidylinositol 3-kinase/Akt pathway promotes translocation of Mdm2 from the cytoplasm to the nucleus. Proc Natl Acad Sci USA 98: 11598-11603, 2001.

49. Greuber EK, Smith-Pearson P, Wang J and Pendergast AM: Role of ABL family kinases in cancer: From leukaemia to solid tumours. Nat Rev Cancer 13: 559-571, 2013.

50. Shaul $\mathrm{Y}$ and Ben-Yehoyada M: Role of c-Abl in the DNA damage stress response. Cell Res 15: 33-35, 2005.

51. Barilá D and Superti-Furga G: An intramolecular SH3-domain interaction regulates c-Abl activity. Nat Genet 18: 280-282, 1998.

52. Greither T, Grochola LF, Udelnow A, Lautenschläger C, Würl P and Taubert H: Elevated expression of microRNAs 155, 203, 210 and 222 in pancreatic tumors is associated with poorer survival. Int J Cancer 126: 73-80, 2010. 